\title{
Impact of Nanoconfinement on Polylactide Crystallization and Gas Barrier Properties
}

\author{
Samira Fernandes Nassar, Nicolas Delpouve, Cyrille Sollogoub, Alain Guinault, Gregory Stoclet, \\ Gilles Régnier, and Sandra Domenek*
}

\begin{abstract}
The barrier properties of poly(L-lactide) (PLLA) were investigated in multinanolayer systems, probing the effect of confinement, the compatibility between the confining and the confined polymer, crystal orientation, and amorphous phase properties. The multilayer coextrusion process was used to confine PLLA between two amorphous polymers (polystyrene, PS; and polycarbonate, PC), which have different chemical affinities with PLLA. Confined PLLA layers of approximately $20 \mathrm{~nm}$ thickness were obtained. The multinanolayer materials were annealed at different temperatures to obtain PLLA crystallites with distinct polymorphs. PLLA annealed in PC/PLLA films at $120{ }^{\circ} \mathrm{C}$ afforded a crystallinity degree up to $65 \%$, and PLLA annealed in PC/PLLA or PS/PLLA films at $85{ }^{\circ} \mathrm{C}$ had a crystallinity degree of $45 \%$. WAXS measurements evidenced that the PLLA lamellas between PS layers had a mixed in-plane and on-edge orientation. PLLA lamellas between PC layers were uniquely oriented in-plane. DMA results evidenced a shift of the PC glass transition toward lower temperature, suggesting the possible presence of an
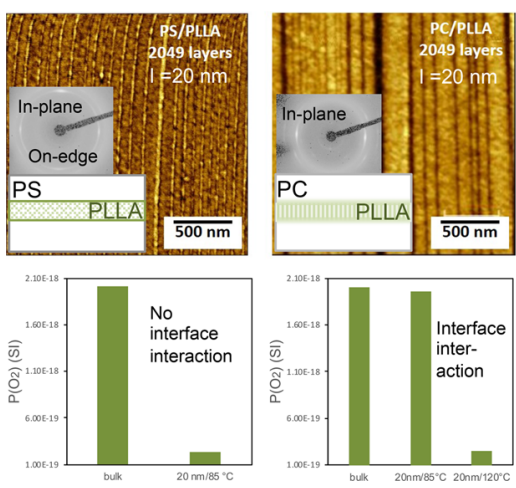
interphase. The development of the rigid amorphous fraction (RAF) in the amorphous phase during annealing was impacted by the confiner polymer. The RAF content of semicrystalline PLLA was about $15 \%$ in PC/ PLLA, whereas it was neglectable in PS/PLLA. The oxygen barrier properties appeared to be governed by RAF content, and no impact of the PLLA polymorph or the crystalline orientation was observed. This study shows that the confinement of PLLA on itself does not impact barrier properties but that the proper choice of the confiner polymer can lead to decrease the phase coupling which creates the RAF. It is the prevention of RAF that decreases permeability.
\end{abstract}

KEYWORDS: PLLA, confinement, interphase, RAF, permeability

\section{INTRODUCTION}

Polylactide (PLA) is today the most produced biodegradable and biobased polymer, mainly used as packaging materials or bioresorbable devices in the biomedical sector. ${ }^{1}$ However, compared with other high-performance polymers, its moderate barrier properties hinder larger-scale commercial application. Optimizing PLA barrier properties receives therefore large academic and industrial interest, as attested by the important number of publications summarized in the reviews of Sonchaeng et $\mathrm{al}^{2}$ Improvements were often achieved by optimizing crystallization, ${ }^{3-8}$ drawing, ${ }^{9}$ and compounding or blending with less-permeable polymers or fillers with high aspect ratio. ${ }^{10,11}$ However, the decrease in barrier properties is rather limited and generally achieved at the expense of other properties (such as optical clarity, degradability, and mechanical toughness). Alternative strategies must therefore be explored.

Confinement of polymers between hard borders by the multilayer coextrusion process is proposed as a promising technology since the report of a spectacular improvement of oxygen barrier properties of poly(ethylene oxide) (PEO) layers crystallized as single crystals in in-plane orientation between polycaprolactone (PCL) layers. ${ }^{12}$ In semicrystalline polymers, crystallization can be profoundly affected by nanoscale confinement: ${ }^{12}$ spherulite growth is greatly hampered, and specific lamellar crystal orientations are created. In particular, it was observed that coextruding PEO, PCL or polypropylene (PP) between hard confining layers led to unique single crystalline lamellas oriented parallel to the layers (inplane). ${ }^{13-17}$ Because the impermeable lamellas with large aspect ratios were oriented perpendicularly to the permeant flux, multiple orders of magnitude reduction in gas permeability was achieved compared with the bulk. The orientation of crystals is thus an important parameter, and many reports exist on the subject. ${ }^{18-23}$ However, results were often behind expectation. ${ }^{10,19,22,23}$ Amorphous-phase dynamics play also a decisive role in the overall barrier properties. In the case of PLA, this was studied in the last years by several 
groups $^{3-5,24}$ and summarized in the recent review of Sonchaeng et al. ${ }^{2}$ However, the impact of confinement of PLA between the hard walls of a second glassy polymer on the barrier properties in such a context has not been studied yet.

Because the control of crystal orientation is crucial for barrier enhancement, a better understanding of the parameters governing the crystalline structuration is needed. ${ }^{25}$ The difficulty lies in the fact that it is not a simple task to split the confiner substrate effect from geometrical effects, as well as the effect of crystal growth kinetics from nucleation. The layer multiplying coextrusion appeared as a relevant tool providing insight into the effect of confinement on the crystallization behavior of polymers. ${ }^{12,16,26}$ Thermal annealing after extrusion of the confined polymer was used to control the orientation habit. In addition to geometrical confinement (i.e., layer thickness), other parameters were found to impact the lamellar crystal orientation, such as the chemical interaction between confining and confined polymers or the annealing conditions. ${ }^{12}$ Besides, with the decrease of the layer thickness, a transition from on-edge lamellas (i.e., lamellas perpendicular to the film layer but polymeric chains parallel to the film layer) to in-plane lamellas (i.e., lamellas parallel to the film layer but polymeric chains perpendicular to the film layer) was observed. A similar transition was found when the (re)crystallization temperature was increased, both on substrate-supported thin films ${ }^{27,28}$ and nanolayered films. ${ }^{21}$ This transition in orientation was attributed to a transition from heterogeneous nucleation to surface nucleation, being deactivated at high temperatures, suggesting that the lamellar orientation is mainly nucleation controlled. 29

The substrate effect is still poorly understood. Enhancing polymer-substrate interaction may impose the polymer chains to orient in layer direction, favoring an on-edge lamellar orientation, but some experimental results on substratesupported thin films contradict this scenario. ${ }^{27}$ The substrate effect on the confined crystallization of PCL in coextruded nanolayered films was investigated, ${ }^{12}$ and it was shown that increased interaction prevented the formation of in-plane crystalline lamellas. As an attempt to understand the substrate effect, $\mathrm{Ma}$ et al. ${ }^{30-32}$ performed dynamic Monte Carlo simulations considering two kinds of interactions with the substrate: sticky walls (related to the case of strong adhesion between polymer and wall) and slippery walls (neutral repulsion between polymer and wall). On-edge lamellas were dominant over the whole range of crystallization temperature and film thickness for slippery walls, while for sticky walls inplane lamellas tended to be dominant.

There are some studies carried out with spin-coated ultrathin PLA films on the influence of annealing temperature on lamellar crystal formation in PLLA. Zhang et al. ${ }^{33}$ showed that the confinement of PLLA between polycarbonate (PC) layers decreased the nucleation and crystal growth rates. Wu et al. ${ }^{34}$ showed that on-edge lamellar crystals of PLLA thin films are mainly formed by cold crystallization at low temperatures, whereas the in-plane crystals occur when the crystallization temperature approaches $100{ }^{\circ} \mathrm{C}$. However, in another study, Maillard and Prud'homme ${ }^{35}$ showed that thin PLLA crystallized at 125 and $160{ }^{\circ} \mathrm{C}$ had on-edge lamellas. The conflicting literature results inform that the main factors influencing the orientation of PLA lamella in thin films are still unknown.

In the present work, we investigate the impact of PLLA confinement between slippery or stick walls by polystyrene (PS) or PC on its crystallization behavior, macromolecular mobility, and gas barrier properties. PS and PLLA are immiscible, ${ }^{36-38}$ and the different surface tensions ${ }^{39}$ of both polymers lead to low compatibility and slippery interfaces. PC and PLA are compatible polymers. Imre et al. ${ }^{40}$ showed that up to approximately $5 \mathrm{wt} \%$ of PC, the $T_{\mathrm{g}}$ of PLLA increased because of the small admixture. The wall of the confiner polymer PC should as a consequence be stickier for PLLA compared with PS walls. In the aim to discriminate interface and confinement effects, the sample set contained (i) 3-layer PLLA/confiner polymer films as a control, (ii) 513-layer PLLA/confiner polymer films as intermediate condition including a largely multiplied number of interfaces but no confinement, and (iii) 2049-layer PLLA/confiner polymer films with a large number of interfaces and confinement of PLLA. The systematic study of geometrical constraints and compatibility effects will help to deduce engineering rules for increasing the performance of the multilayer materials.

\section{MATERIALS AND METHODS}

Materials and Processing. Two couples of polymers were used in this study: polylactide (PLLA)/polystyrene (PS) (PS/PLLA) and polylactide (PLLA)/polycarbonate (PC) (PC/PLLA). PLLA pellets were purchased from Corbion Purac (PLA REVODE 190). The content of L.L-lactide was higher than $99 \mathrm{~mol} \%$. The weight-average molar mass was measured by GPC (Agilent $220 \mathrm{HT}$ ) using PS standards. It was equal to $M_{w}=177 \mathrm{~kg} \mathrm{~mol}^{-1}$ and the dispersity $Đ$, defined as $M_{w} / M_{n}$, was 1.64. The gyration radius of PLLA estimated from the plot of Fang et al. ${ }^{41}$ is around $20 \mathrm{~nm}$. Polycarbonate (PC) pellets were obtained from Gazechim (PC121R). Its characteristics were $M_{\mathrm{n}}=19000 \mathrm{~g} / \mathrm{mol} ; M_{\mathrm{w}}=43000 \mathrm{~g} / \mathrm{mol} ; Ð=2,4$ (done in THF, standards PS). Polystyrene (PS) pellets were purchased from Total Petrochemicals (PS 1340). Its characteristics were $M_{\mathrm{n}}=112000 \mathrm{~g} /$ mol; $M_{\mathrm{w}}=245000 \mathrm{~g} / \mathrm{mol} ; Ð=2,2$ (done in THF, standards PS).

A multilayer coextrusion process was used to elaborate multilayered films composed of 3, 513, and 2049 alternating layers of PLLA and PS or PC. The composition of the multilayer films was $75 \mathrm{wt} \%$ confiner polymer and 25 wt \% PLLA. In brief, PLLA and PC was dried using a SOMOS dryer (France) with circulation of dry air overnight. The residual humidity of PLLA amounted to 250 to $300 \mathrm{ppm}$ and that of PC was between 150 and $250 \mathrm{ppm}$. PS was used as received. The experimental setup consisted of two single screw extruders (Rheoscam Scamex, France) of $20 \mathrm{~mm}$ with gear pumps, a three-layer feed block (A-B-A), a series of layer-multiplying elements, a flat die, and chill rolls (Scamex, France), as described by our earlier work. ${ }^{42}$ The layermultiplying elements cut the flow in half vertically and subsequently superpose, compress, and stretch it to its original width. A series of $n$ elements leads thus to $2^{n+1}+1$ alternating layers. The extrusion conditions are given in the Supporting Information S.1. In order to ensure the best homogeneity of the layers and to avoid layer breakup, the viscosity ratio should be as close as possible to $1{ }^{43}$ in the shear rate range of coextrusion process (typically between 1 and $50 \mathrm{~s}^{-1}$ ). In the case of PC/PLLA, even if the PC grade chosen for this study had a low viscosity, the achieved viscosity ratio in the processing window was around 0.35 . In the case of PS/PLLA, a viscosity ratio of 1 was obtained. A graph containing the experimental data is given in the Supporting Information S.2.

In order to obtain films with different nominal individual PLLA layer thickness, the chill roll speed and the number of mixing elements were varied. The target total film thickness to reach a given nominal PLLA layer thickness was calculated taking into account the weight percentage of PLLA and the number of PLLA layers $\left(l_{\mathrm{PLLA}}=\right.$ $0.25 * l_{\text {film }} /$ nPLLA layers $)$. Using 8 multiplying elements (256 PLLA layers), the total target film thickness is $300 \mu \mathrm{m}$ and the nominal individual PLLA layer thickness is $300 \mathrm{~nm}$. Using 10 multiplying elements (1024 PLLA layers), the total target film thickness is $80 \mu \mathrm{m}$, and the nominal individual PLLA layer thickness $20 \mathrm{~nm}$. Different processing conditions were tested. The following optimized process conditions were employed to reach the target total film thickness 
using PS as confiner polymer: 8 multiplying elements and a chill roll of speed $60 \mathrm{~cm} / \mathrm{min}$, and 10 multiplying elements and a chill roll speed $200 \mathrm{~cm} / \mathrm{min}$. The conditions using PC as confiner polymer were the following: 8 multiplying elements and a chill roll of speed 60 $\mathrm{cm} / \mathrm{min}$, and 10 multiplying elements and a chill roll speed of 180 $\mathrm{cm} / \mathrm{min}$. The target total film thickness was reached with an experimental variability of approximately $10 \%$. The exact PLLA layer thickness was calculated for each sample taking into account the measured total film thickness. In the aim to simplify the sample nomenclature, we used the nominal layer thickness in the text. To test for variability in oxygen barrier properties caused by changing processing conditions, a broad sample set was used, including the samples produced during process optimization. All corresponding data are shown in Supporting Information S.5.

The crystallization of the PLLA layer was performed subsequently to extrusion, thanks to thermal treatments using a heating press (Darragon, France) to avoid deformation of films due to internal stress release. Rectangular samples of approximately $15 \times 20 \mathrm{~cm}$ were cut from extruded films, discarding the edges to avoid thickness differences. They were stored in a desiccator over $\mathrm{P}_{2} \mathrm{O}_{5}$. The samples were then sandwiched between two steel plates lined inside with two Teflon sheets. They were hot-pressed at 85,100 , or $120{ }^{\circ} \mathrm{C}$ for 180 min at a pressure of $5 \times 10^{6} \mathrm{~Pa}$ and cooled under air to room temperature.

Analysis Methods. Atomic Force Microscopy (AFM). AFM was used to characterize the layered morphology of the coextruded films. AFM images were obtained in tapping mode using a multimode microscope controlled by a Veeco Nanoscope V controller (France). The tips (silicon, spring constant $40 \mathrm{~N} / \mathrm{m}$, oscillation frequency ca. $300 \mathrm{kHz}$ ) had a radius of curvature less than $10 \mathrm{~nm}$. Specimens were taken from the center of the extruded films and were cut from the cross section with an ultramicrotome 2088 Ultrotome V (LKB, France) equipped with a diamond knife at a cutting speed of $1 \mathrm{~mm}$. $\mathrm{s}^{-1}$. Fifteen images were recorded at full resolution $(4096 \times 4096$ pixels) with a scan rate of $0.9 \mathrm{~Hz}$ throughout the thickness of the film. Following the method developed by Bironeau et al., ${ }^{44}$ the analysis of these 15 images containing each around 20 layers was representative of the whole sample with an variability of $10 \%$.

Wide-Angle X-ray Scattering (WAXS). WAXS data were recorded with the help of a PANanalytical diffractometer (France). The parameters were a beam at $40 \mathrm{kV}$ and $20 \mathrm{~mA}$. The $\mathrm{Cu} \mathrm{K} \alpha$ radiation $(\lambda$ $=1.54 \AA$ ) was chosen with a Nickel filter. The WAXS diffractograms were recorded by a CDD camera (Photonic Science, France) in 2-D. WAXS data of crystallization kinetics were recorded with to a Genix microsource (XENOCS, France) equipment operating at $50 \mathrm{kV}$ and 1 $\mathrm{mA}$. The $\mathrm{Cu} \mathrm{K} \alpha$ radiation used $(1=1.54 \AA$ A $)$ was selected with a curved mirror monochromator. The annealing of the samples was done with a heating plate (Linkam) in situ in the X-ray diffraction machine. Two or three films were stacked in order to increase the WAXS signal and then introduced in a heating stage and heated from the bottom side. Before analysis, standard corrections were applied to the patterns such as dark current subtraction and background correction. The quantification of the crystallinity degree was obtained by subtraction of the signal of the area of the amorphous halo from the total surface area of the diffractogram.

The isothermal crystallization kinetics of PLLA was analyzed with the help of the Avrami equation:

$$
\alpha(t)=1-\exp \left(-K \cdot t^{n}\right)
$$

where $\alpha(t)$ is the relative crystallinity fraction at time $t$ calculated as $\alpha(t)=$ Surface_Peak $(t) /$ Surface_Peak $\left(t_{\text {end }}\right) . K$ is the crystallization rate constant, and $n$ is the Avrami exponent depending on the nucleation and the growth geometry (sphere, disc, etc.).

Modulated Temperature Differential Scanning Calorimetry (MTDSC). The MT-DSC analyses were performed on a Thermal Analysis Instrument DSC Q100 (TA Instruments, France). Nitrogen was used as the purge gas $\left(50 \mathrm{~mL} \cdot \mathrm{min}^{-1}\right)$. Amorphous samples were cut from the extruded films and annealed samples from the hot-pressed films. The sample mass was approximately 5-10 mg. The sample holders were Tzero hermetic aluminum pans. Calibration in temperature and enthalpy was carried out using an Indium standard. Calibration in heat capacity was performed using sapphire as a reference. Heat-only temperature modulation method (oscillation amplitude of $0.32 \mathrm{~K}$, oscillation period of $60 \mathrm{~s}$, and heating rate of $2 \mathrm{~K} \cdot \mathrm{min}^{-1}$ ) was applied to get the content of crystalline and amorphous fractions. The glass transition region of PLLA was specifically investigated using a heatcool temperature modulation (oscillation amplitude of $3 \mathrm{~K}$, oscillation period of $120 \mathrm{~s}$, and heating rate of $1 \mathrm{~K} \cdot \mathrm{min}^{-1}$ ). Reversing and nonreversing heat flows were obtained from the deconvolution procedure proposed by Reading and coauthors. ${ }^{45}$ An example is shown in Supporting Information S.3.

Dynamic Mechanical Thermal Analysis (DMTA). DMTA was carried out on thin film samples $(10.25 \mathrm{~mm}$ length, $5.00 \mathrm{~mm}$ width, and a thickness between 0.08 and $0.12 \mathrm{~mm}$ ) using a Triton Tritec (France) apparatus operating in dynamic tensile mode. The frequency was set at $1 \mathrm{~Hz}$ and the dynamic displacement at $10 \mu \mathrm{m}$. The samples were heated from 25 to $200{ }^{\circ} \mathrm{C}$ at a heating rate of $2{ }^{\circ} \mathrm{C} \cdot \mathrm{min}^{-1}$.

Oxygen Permeability. The oxygen transmission rate was measured with a Systech analyzer 8001 (France) or a Brugger GDP-C (Germany) at $23{ }^{\circ} \mathrm{C}$ and $0 \% \mathrm{RH}$. Comparative tests on the same samples were done to ensure the equivalence of the results. Oxygen permeability was calculated from the measured oxygen transmission rate (OTR) by multiplying it by the sample thickness (measured with a micrometer on 9 points). For assessing reproducibility, permeability measurements were carried out on at least two different samples obtained by the same protocol. In the Supporting Information S.5, all raw data used for the discussion of the barrier properties are given, including processing conditions, measured total film layer thickness, and calculated nominal PLLA layer thickness.

The permeability of PLLA was calculated using the eq 2 below, derived from the series model for multilayered assemblies. In this equation, $75 \%$ by weight of PS or PC and $25 \%$ by weight of PLLA are considered:

$$
\frac{1}{P_{\text {film }}}=\frac{0.25}{\rho_{\text {PLLA }} \cdot P_{\text {PLLA }}}+\frac{0.75}{\rho_{\text {confiner }} \cdot P_{\text {confiner }}}
$$

where PLLA density $\left(\rho_{\text {PLLA }}\right)$ is $1.25 \mathrm{~g} / \mathrm{cm}^{3}$, PS density $\left(\rho_{\text {PS }}\right)$ is $1.05 \mathrm{~g} /$ $\mathrm{cm}^{3}$, and PC density $\left(\rho_{\mathrm{PC}}\right)$ is $1.2 \mathrm{~g} / \mathrm{cm}^{3}$.

\section{RESULTS AND DISCUSSION}

Multinanolayer Morphology of PC/PLLA and PS/PLLA Samples. The layer morphology of the sample set, which was achieved by multinanolayer coextrusion process, was verified by means of AFM imaging. Figure 1 shows AFM images for PC/PLLA and PS/PLLA samples using 10 mixing elements (2049 layers). The AFM pictures using 8 mixing elements (513 layers) are shown in the Supporting Information S.4. PC and PS appear bright while PLLA appears dark. The observed PLLA layers of the amorphous samples (Figure 1a,d) were continuous even if some thickness heterogeneities were observed. The PC/PLLA samples (Figure 1d) were more heterogeneous compared with PS/PLLA, probably because of a viscosity ratio of both polymers in the range of 0.3 (Supporting Information S.2). The acquisition of AFM images of PC/PLLA was challenging because of very low contrast and high polarity leading to quick pollution. Few images were obtained, which prevented a complete statistical analysis of the sample. On the basis of a sampling composed of several tens of layers, the measured mean thickness, reported in Table 1, was in a reasonable agreement with the expected nominal thickness. PLLA crystallizes in the $\alpha^{\prime}$-form at temperatures lower than $90{ }^{\circ} \mathrm{C}$ and in the $\alpha$-form at temperatures higher than $110{ }^{\circ} \mathrm{C}$. At intermediate temperatures, a mixture of both polymorphs is obtained. ${ }^{1}$ To test the effect of the polymorph on the properties of PLLA, low-temperature annealing of PS/ PLLA and PC/PLLA at $85{ }^{\circ} \mathrm{C}$ was carried out. The high- 

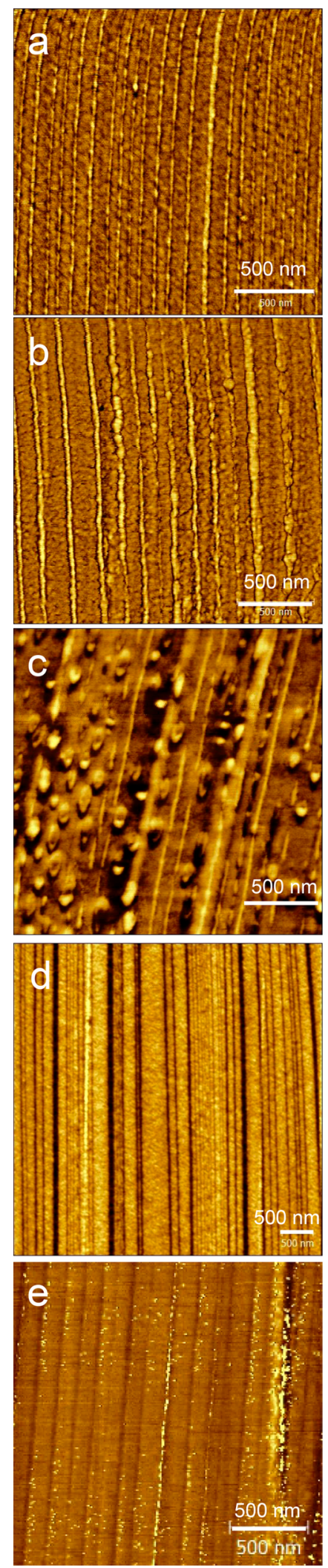

Figure 1. Tapping mode AFM phase image of nanolayered PS/PLLA and PC/PLLA containing 2049 layers. (a) Amorphous PS/PLLA, (b) annealed PS/PLLA $85{ }^{\circ} \mathrm{C}$, (c) annealed PS/PLLA $100{ }^{\circ} \mathrm{C}$, (d) amorphous PC/PLLA, (e) annealed PC/PLLA $120^{\circ} \mathrm{C}$.

temperature annealing of PLLA was limited by the glass transition of PS $\left(T_{\mathrm{g}}=97{ }^{\circ} \mathrm{C}\right)$. We tested an annealing
Table 1. PC/PLLA and PS/PLLA Multilayered Films Used to Study the Effect of PLLA Layer Thickness on the Confinement Effect

\begin{tabular}{cccccc} 
multipliers & $\begin{array}{c}\text { target } \\
\text { film } \\
\text { number } \\
\text { of layers } \\
\text { thickness } \\
(\mu \mathrm{m})\end{array}$ & $\begin{array}{c}\text { PLLA } \\
\text { nominal } \\
\text { thickness } \\
(\mathrm{nm})\end{array}$ & $\begin{array}{c}\text { Measured } \\
\text { PLLA } \\
\text { thickness in } \\
\text { PC }(\mathrm{nm})\end{array}$ & $\begin{array}{c}\text { Measured } \\
\text { PLLA } \\
\text { thickness in } \\
\text { PS }(\mathrm{nm})^{42}\end{array}$ \\
0 & 3 & 120 & 30000 & & \\
8 & 513 & 300 & 300 & $330 \pm 50$ & $220 \pm 50$ \\
10 & 2049 & 80 & 20 & $22 \pm 5$ & $20 \pm 5$ \\
\hline
\end{tabular}

temperature at $100{ }^{\circ} \mathrm{C}$, in the aim to avoid complete devitrification of the PS layers. Typical AFM pictures of annealed PS/PLLA samples are shown in Figure 1b,c. The crystallization of the PLLA layers at $85{ }^{\circ} \mathrm{C}$ (Figure $1 \mathrm{~b}$ ) seemed to induce some supplementary heterogeneities of the layer thickness, but no layer breakup was observed. On the contrary, layer breakup and formation of PLLA droplets were observed after annealing at $100{ }^{\circ} \mathrm{C}$ (Figure 1c). We attribute this result to devitrification of PS during the annealing treatment, because the temperature control of the heating press is not very precise and at the beginning of the experiment, heating overshoots can occur which might have increased the effective temperature above $T_{\mathrm{g}}$ of PS. The annealing of PC/PLLA was carried out at temperatures of 85 and $120{ }^{\circ} \mathrm{C}$. The $T_{\mathrm{g}}$ of $\mathrm{PC}$ equals to 144 ${ }^{\circ} \mathrm{C}$; therefore, no layer breakup was suspected. However, because of the better compatibility of PC and PLLA, only very little contrast exists. Therefore, correct AFM pictures of the annealed systems were very difficult to obtain. Only some pictures of annealed PC/PLLA at $120^{\circ} \mathrm{C}$ were caught (Figure 1e). We suppose that, in analogy to the PC/PLLA annealed at $120{ }^{\circ} \mathrm{C}$ and PS/PLLA annealed at $85{ }^{\circ} \mathrm{C}$, no layer breakup occurred in the PC/PLLA samples during annealing at $85^{\circ} \mathrm{C}$.

Table 1 presents the measured achieved layer thickness of PC/PLLA and PS/PLLA films as a function of extrusion setup.

Crystallization Kinetics and Crystalline Morphology Confined PLLA Layers. The crystallization kinetics of PLLA under confinement were investigated using in situ WAXS experiments on stacked films. The PS/PLLA system was already presented in our earlier paper. ${ }^{42}$ For the sake of comparison, the numerical data are shown together with the analysis of the PC/PLLA system in Table 2. The crystallization

Table 2. Parameters of Isothermal Crystallization with Avrami Model $^{a}$

$\begin{array}{lccc} & t_{1 / 2}(\mathrm{~h}) & n & K\left(\mathrm{~h}^{-n}\right) \\ \text { PLLA bulk } 85{ }^{\circ} \mathrm{C} & 0.31 & 3 & 21.83 \\ \text { PLLA bulk } 120{ }^{\circ} \mathrm{C} & 0.1 & 3 & 11436 \\ \text { PS/PLLA } 20 \mathrm{~nm} 85{ }^{\circ} \mathrm{C}^{42} & 1.84 & 1.7 & 0.25 \\ \text { PC/PLLA } 20 \mathrm{~nm} 85{ }^{\circ} \mathrm{C} & 1.54 & 2 & 0.28 \\ \text { PC/PLLA } 20 \mathrm{~nm} 120{ }^{\circ} \mathrm{C} & 0.15 & 2 & 19.10\end{array}$

${ }^{a} t_{1 / 2}$ : half time; $n$ : Avrami exponent; K: Avrami crystallization rate constant, data of PS/PLLA $20 \mathrm{~nm}$ are repeated from our earlier publication, ref 42 .

kinetics of PLLA between PC layers at 85 and $120{ }^{\circ} \mathrm{C}$ are plotted in Figure 2. PLLA crystallization under confinement at $85{ }^{\circ} \mathrm{C}$ evolves slower as compared with kinetics of the bulk. This result corresponds to the result obtained under confinement of PLLA in PS. ${ }^{42}$ Here, we show that the same behavior is also observed at high crystallization temperature (i.e., $120^{\circ} \mathrm{C}$ ). 


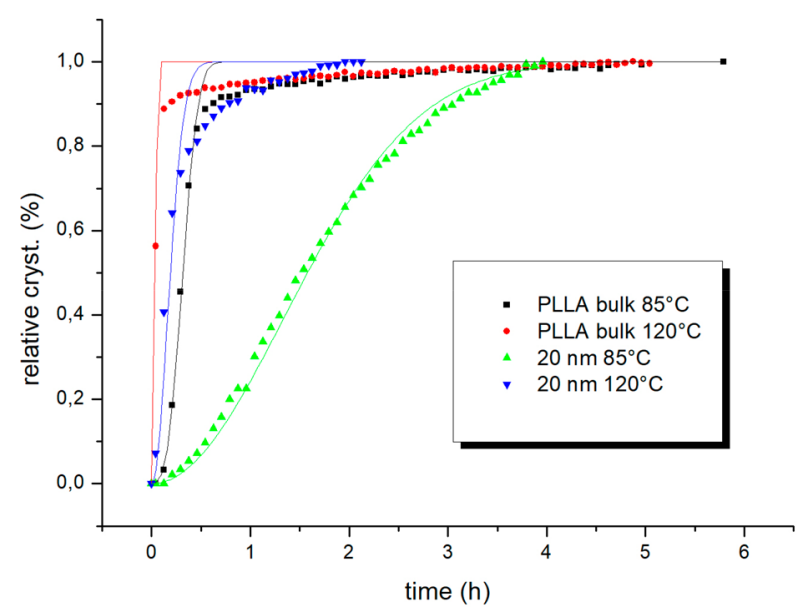

Figure 2. Relative crystallinity and Avrami model (continuous lines) versus crystallization time for isothermal crystallization for bulk PLLA and $20 \mathrm{~nm}$ samples at 85 and $120^{\circ} \mathrm{C}$.

The kinetic parameters were modeled with the help of the Avrami theory. The Avrami exponent and the crystallization rate constant were retrieved from the slope and intercept of the linearized crystallization kinetics. While the Avrami exponent of the bulk samples was close to 3, it was somewhat lower than 2 for the confined samples, which approximately corresponds to a two-dimensional crystallization growth with an instantaneous nucleation. The calculated crystallization kinetics (eq 1) are superposed on the experimental data in Figure 2, setting the value of the Avrami constant to 3 for the bulk samples and to 2 for the confined ones. For bulk PLLA and the confined film annealed at $120{ }^{\circ} \mathrm{C}$, nonlinear behavior was observed in the double-logarithmic curve of the Avrami plot $(\ln (-\ln (1-$ $a(t)))$ vs $\ln (t)$, not shown), which suggested the existence of a secondary crystallization. ${ }^{46}$ The annealing at $120{ }^{\circ} \mathrm{C}$, that is, close to melting temperature, indeed favored the apparition of the secondary crystallization because macromolecules are more mobile. ${ }^{46,47}$ The Avrami model of $20 \mathrm{~nm}$ thick PLLA annealed at $85{ }^{\circ} \mathrm{C}$ fitted well, and its double-logarithmic curve $(\ln (-\ln (1-a(t)))$ vs $\ln (t))$ was linear. No secondary crystallization was observed. The Avrami parameters of the bulk samples are consistent with literature data. ${ }^{48}$

The half-time of crystallization $\left(t_{1 / 2}\right)$ is related to the $n$ and $K$ parameters and was defined as the time at which the extent of relative crystallization reached a value of $50 \%$. Similarly to the results obtained for PLLA confined by PS, half-time crystallization for PLLA confined by PC increased compared with bulk PLLA at $85{ }^{\circ} \mathrm{C}$. $\mathrm{K}$ was strongly dependent on the annealing temperature and increased at $120{ }^{\circ} \mathrm{C}$, which was expected from the known behavior of PLLA crystallization in bulk $^{48}$ (Table 2).

Figure 3a shows the WAXS intensity profile of the PS/PLLA annealed samples. The extruded samples showed an amorphous halo. The strong reflections of the PLLA crystallites attributed to the $(200) /(110)$ and (203) planes could be observed in samples annealed at $85{ }^{\circ} \mathrm{C}$. Depending on the crystallization temperature, PLLA can crystallize into two distinct polymorphs. Particularly, a low crystallization temperature (i.e., $85^{\circ} \mathrm{C}$ ) favors the $\alpha^{\prime}$-polymorph, while a high temperature (i.e., $120{ }^{\circ} \mathrm{C}$ ) leads to the $\alpha$-form. ${ }^{49-51}$ These crystalline forms $\alpha$ and $\alpha^{\prime}$ can be differentiated in the WAXS patterns. A small shift in $2 \theta$ values of the two strongest reflections to smaller angles and the appearance of a small reflection at $2 \theta=24.8^{\circ}$ is characteristic of the $\alpha^{\prime}$-form. ${ }^{52}$ However, because of the small amount of PLLA in the multilayer samples, the minor reflections characteristics of $\alpha^{\prime}$ form at $2 \theta=24.8^{\circ}$ were not observed. Therefore, the presence of the $\alpha^{\prime}$ polymorph of PLLA, although likely, cannot be evidenced in PS/PLLA films. Figure $3 \mathrm{~b}$ shows the WAXS results of PC/PLLA samples. Extruded samples exhibit uniquely an amorphous halo. The annealed films at 85 and $120{ }^{\circ} \mathrm{C}$ during $180 \mathrm{~min}$ show peaks corresponding to the crystallites of PLLA. The strong reflections of PLLA attributed to the $(110) /(200)$ and (203) planes could be observed in both annealed samples. The strong reflections were shifted to smaller angles in the films annealed at $85{ }^{\circ} \mathrm{C}$, and a very small signal at $2 \theta=24.8^{\circ}$ was observed. The annealing of the samples PC/PLLA samples at $85{ }^{\circ} \mathrm{C}$ yielded thus PLLA crystals in $\alpha^{\prime}$-form and the annealing at $120^{\circ} \mathrm{C}$ gave rise to the $\alpha$-polymorph.

The orientation of crystalline lamellas and the crystalline polymorph of extruded and annealed PS/PLLA and PC/PLLA multilayer films were analyzed by WAXS. The WAXS patterns of PS/PLLA films are shown in Figure 4. No arc reflections of crystalline structures were observed in the extruded samples (Figure $4 a-c$ ), as they are amorphous. WAXS patterns of 30 $\mu \mathrm{m}$ and $300 \mathrm{~nm}$ PS/PLLA films after annealing at $85{ }^{\circ} \mathrm{C}$ exhibited isotropic reflection rings from $(110) /(200)$ plane of PLLA (Figure 4d,e). In the case of PS/PLLA $20 \mathrm{~nm}$, crystallization at $85{ }^{\circ} \mathrm{C}$ resulted in crystallization forming
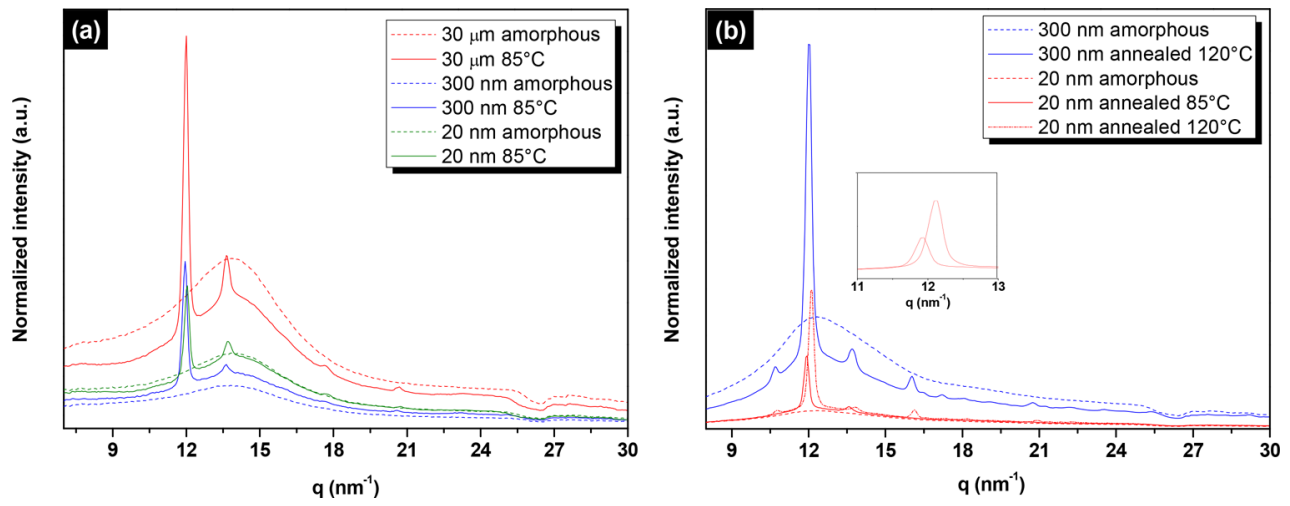

Figure 3. Normalized WAXS intensity profiles of amorphous and annealed PLLA (annealing temperatures 85 and $120{ }^{\circ} \mathrm{C}, 180$ min) as a function of the PLLA layer thickness $(30 \mu \mathrm{m}, 300 \mathrm{~nm}, 20 \mathrm{~nm}$ ): (a) PS/PLLA system (b) PC/PLLA system. The plot shows an enlarged image of the (110)/ (200) plan. 


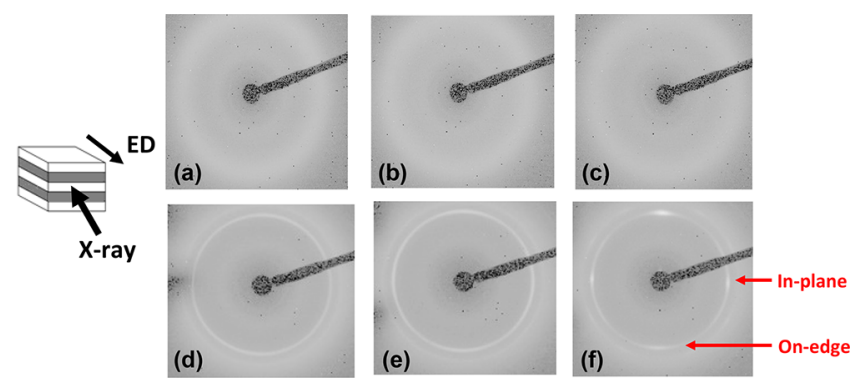

Figure 4. WAXS patterns obtained in extrusion direction (ED) for PS/PLLA (a) $30 \mu \mathrm{m}$. (b) $300 \mathrm{~nm}$ and (c) $20 \mathrm{~nm}$ after extrusion and (d) $30 \mu \mathrm{m},(\mathrm{e}) 300 \mathrm{~nm}$, and (f) $20 \mathrm{~nm}$ after annealing at $85^{\circ} \mathrm{C}$ during $180 \mathrm{~min}$

lamellas mainly oriented in both directions, on-edge and inplane (Figure $4 \mathrm{f})$. The presence of on-edge lamellas was confirmed by meridional reflections for $(110) /(200)$ crystal plane, while in-plane crystals led to the formation of equatorial arcs for the same crystal plane. The formation of mixed lamellas has already been observed on other multilayered films like polypropylene confined by PC ${ }^{17}$ or PCL confined by PS/ $\mathrm{PP}^{53}$

The WAXS patterns of PC/PLLA films are shown in Figure 5. The extruded films were amorphous. In PC/PLLA films
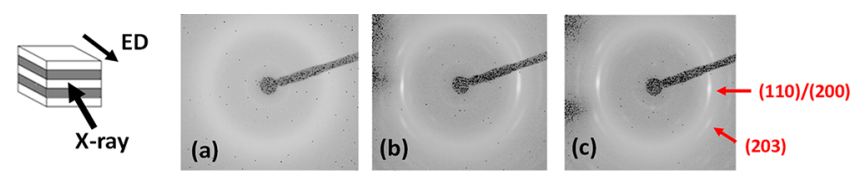

Figure 5. WAXS patterns obtained in extrusion direction (ED) for PC/PLLA samples (a) $20 \mathrm{~nm}$ after extrusion, (b) $20 \mathrm{~nm}$ after annealing at $85^{\circ} \mathrm{C}$ during $180 \mathrm{~min}$, and (c) $20 \mathrm{~nm}$ after annealing at $120^{\circ} \mathrm{C}$ during $180 \mathrm{~min}$.

with $20 \mathrm{~nm}$ PLLA thickness and annealed at 85 and $120{ }^{\circ} \mathrm{C}$, the equatorial reflections for $(110) /(200)$ crystal plane were observed, which indicated the presence of in-plane lamellas. Another evidence for in-plane lamellas in PC/PLLA samples was the reflections at about $30^{\circ}$ for the (203) crystal plane. For this crystal plane with in-plane lamellas, and since PLLA has an orthorhombic form ${ }^{54,55}$ with parameters $a=1.06 \mathrm{~nm}, b=$ $0.610 \mathrm{~nm}$, and $c=2.88 \mathrm{~nm},{ }^{56}$ the beam hits lamellas at $60^{\circ}$, reflecting an arc at about $30^{\circ}$.

In conclusion, the confiner polymer influenced apparently the crystalline orientation of PLLA under confinement. Confining PLLA between PS layers induced a mixed orientation of in-plane and on-edge crystals, while PC layers resulted in an in-plane orientation. The same result was obtained notwithstanding the crystallization temperature. The on-edge orientation was observed by different authors as being linked to low crystallization temperatures and homogeneous nucleation, ${ }^{25,29,34}$ while in-plane orientation was observed at higher temperatures and in the case of heterogeneous nucleation. ${ }^{29}$ At intermediate temperatures, both types of orientation can coexist, which might be the case in the PS/ PLLA system. The transition region for the in-plane to on-edge lamellas' orientation was also observed to shift toward lower temperatures for a more interactive system. ${ }^{12}$ This may explain why, for similar crystallization temperature $\left(T_{c}=85^{\circ} \mathrm{C}\right)$, the more interactive system PC/PLLA leads to only in-plane lamellas, while mixed orientated crystals are present for the noninteracting substrate PS. The higher compatibility of PC with PLLA might facilitate heterogeneous nucleation leading to in-plane crystals.

Microstructure Quantification and Properties of the Amorphous Phases of Confined PLLA. The quantification of crystalline and amorphous fractions in multilayer films from classical DSC needs caution because of the possible superimposition of thermal events in the same temperature domain. This problem can be partly solved by MT-DSC which allows separating two signals, respectively named reversing and nonreversing, the events that only related to the heat capacity change, such as the glass transition, and the kinetic events such as crystallization. Figure 6 shows sample data of the analysis of the films with $20 \mathrm{~nm}$ PLLA layer thickness. Figure 6a shows the reversing and nonreversing heat flows of the PS/PLLA film on the example of the amorphous sample with $20 \mathrm{~nm}$ layer thickness. The PLLA cold crystallization was partly superposed on the PS glass transition. Likewise, Figure $6 \mathrm{~b}$ shows that the glass transition of $\mathrm{PC}$ is located in the temperature domain where cold crystallization of PLLA ends and melting starts. Therefore, the use of MT-DSC offers a better picture of the glass transition of both confiner and confined polymers.

In the first step, the impact of the both the layer thickness and the PLLA microstructure on the confiner polymer has been assessed. Table 3 contains the quantitative data. Figure $6 \mathrm{c}$ shows the zoomed-in graph on the PS glass transition in $20 \mathrm{~nm}$ thickness PLLA films. One can observe that the signature of PS glass transition appears noisier when PLLA is amorphous, whereas it is well-defined when PLLA is crystallized at $85^{\circ} \mathrm{C}$, probably because the crystals help to limit the stress relaxation. Independently of PLLA layer thickness and microstructure, no change in the glass transition temperature of PS was observed, evidencing immiscibility between the two polymers (Table 3 ). According to Table 3, the layer thickness of amorphous PLLA has no obvious influence on the PC glass transition, but Figure $6 \mathrm{~d}$ shows that the annealing of $20 \mathrm{~nm}$ thick PLLA layers induces a broadening of the glass transition. Moreover, when the annealing was performed at $120{ }^{\circ} \mathrm{C}, \mathrm{T}_{\mathrm{g}}$ of PC slightly decreased. The increase of the layer number caused the increase of the surface area between both polymers, which would enable us to detect the partial miscibility between both polymers. Besides, the annealing at high temperature facilitates the diffusion of one layer into another. The compatibility of PS and PLLA is supposed to be lower than the compatibility between PC and PLLA, which can show partial miscibility. ${ }^{40}$ Our hypothesis is that PC interacts strongly enough with PLLA to create an interphase. Another way to evaluate the interpenetration between confiner and confined polymers consists in the determination of the confiner heat capacity step $\Delta C_{\mathrm{p}}$ at $T_{\mathrm{g}}$, then to compare it with the value for a bulk polymer, $\Delta C_{\mathrm{p}, \text { bulk }}$. Because the composition of the multilayer films is $75 \mathrm{wt} \%$ confiner polymer and $25 \mathrm{wt} \%$ PLLA, one expects $\Delta C_{\mathrm{p}} / \Delta C_{\mathrm{p}, \text { bulk }}$ to be close to $75 \%$ in the absence of interpenetration, while it would be lower than $75 \%$ in the presence of interpenetration. Indeed, the existence of an interphase implies that a part of PC devitrifies between both glass transitions of bulk PC and PLLA. This calculation gives reliable results because both confiners are amorphous. As shown in Table 3, $\Delta C_{\mathrm{p}} / \Delta C_{\mathrm{p}, \text { bulk }}$ is most often close to, and never below, $75 \%$ in PS/PLLA films. The results are more dispersed in the case of PC/PLLA multilayer films. As a consequence, interactions between PC and PLLA are likely, 

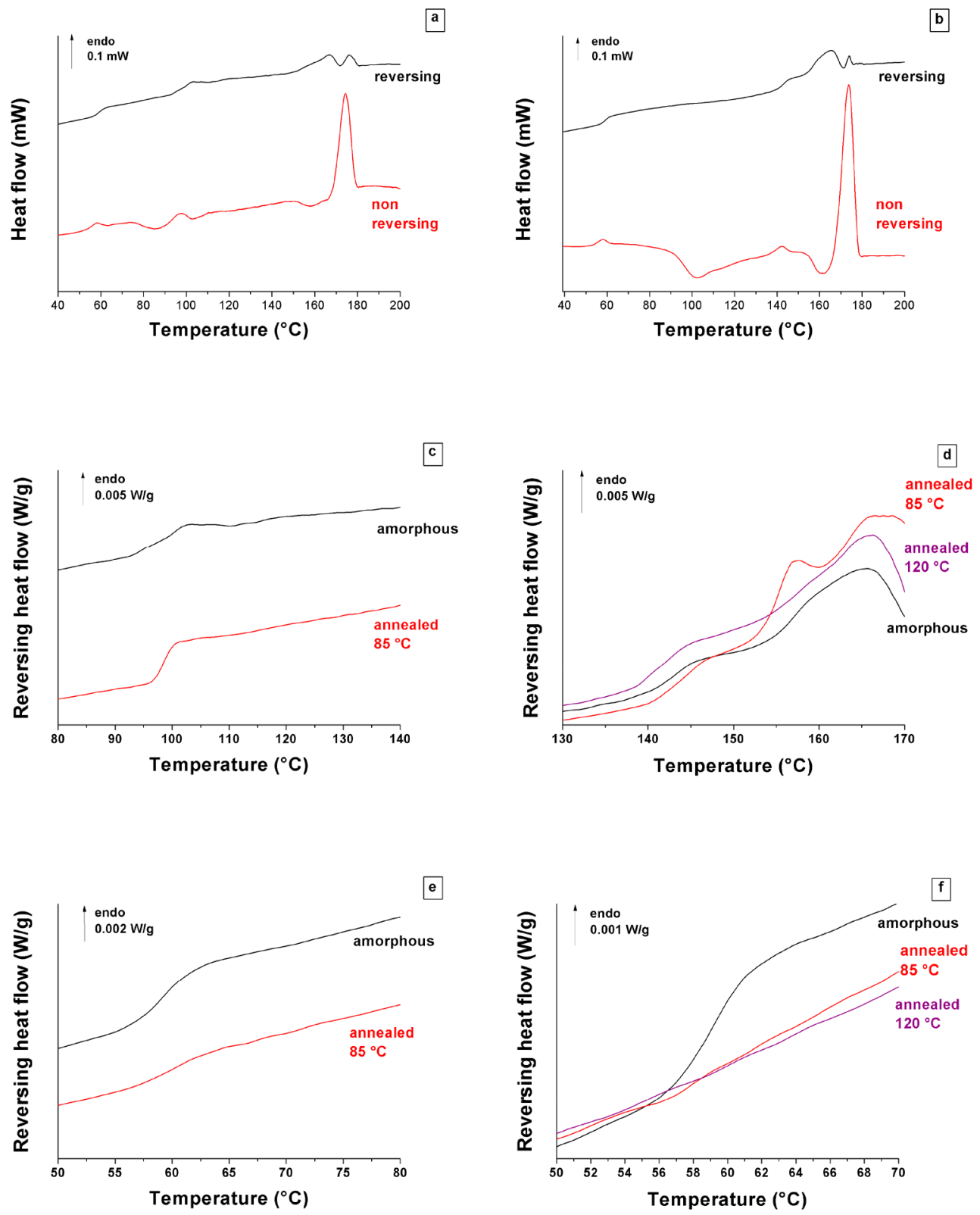

Figure 6. MT-DSC analysis of amorphous and annealed PS/PLLA (left column) and PC/PLLA films with 20 nm thickness (right column). (a) Reversing and nonreversing heat flow of amorphous PS/PLLA $\left(85^{\circ} \mathrm{C}\right)$ recorded with the heat-only protocol; (b) Reversing and nonreversing heat flow of amorphous PC/PLLA $\left(85{ }^{\circ} \mathrm{C}\right.$ ) recorded with the heat-only protocol; (c) Zoomed-in graph of the glass transition region of PS in amorphous and annealed PS/PLLA films recorded with the heat-only protocol; (d) Zoomed-in graph of the glass transition region of PC in amorphous and annealed PC/PLLA films recorded with the heat-only protocol; (e) Zoomed-in graph of the glass transition region of PLLA in amorphous and annealed PS/PLLA films recorded with the heat-cool protocol; (f) Zoomed-in graph of the glass transition region of PLLA in amorphous and annealed PC/PLLA films recorded with the heat-cool protocol.

but the assumption of an interphase can neither be confirmed nor refuted at this stage.

The impact of confinement on the PLLA microstructure was analyzed with the help of the three-phase model, as previously reported. ${ }^{4,42}$ During crystallization, PLLA can develop a rigid amorphous fraction (RAF) in the amorphous phase, which describes coupling between crystalline and amorphous phases by tie chains. ${ }^{3,4,42,47,57-59}$ The RAF does not relax at the glass transition of the polymer and is therefore not captured by the height of the heat capacity step at $T_{\mathrm{g}}$.

The microstructure of the polymer can thus be quantified by

$$
\begin{gathered}
X_{\mathrm{c}}+X_{\mathrm{MAF}}+X_{\mathrm{RAF}}=1, \text { with } X_{\mathrm{MAF}}=\frac{\Delta C_{\mathrm{p}}}{\Delta C_{\mathrm{p}}^{0}} \\
\text { and } X_{\mathrm{c}}=\frac{\Delta H_{\mathrm{m}}-\Delta H_{\mathrm{cc}}}{\Delta H_{\mathrm{m}}^{0}}
\end{gathered}
$$

where $X_{\mathrm{MAF}}$ is the content of the mobile (bulk-like) amorphous fraction (MAF) in the amorphous phase, $X_{c}$ the crystallinity degree, $\Delta C_{\mathrm{p}}$ the heat capacity change at glass transition, $\Delta C_{\mathrm{p}}^{0}$ the heat capacity change at glass transition of the fully amorphous sample, $\Delta H_{\mathrm{m}}$ the melting enthalpy, $\Delta H_{\mathrm{cc}}$ the enthalpy of the cold crystallization, and $\Delta H_{\mathrm{m}}{ }^{0}$ the melting enthalpy of the perfect crystal at infinite size $\left(93.1 \mathrm{~J} / \mathrm{g}^{60}\right)$. All 
Table 3. Glass Transition Temperature $\left(T_{\mathrm{g}}\right)$ of PS and PC in Multinanolayers Films

\begin{tabular}{|c|c|c|c|}
\hline & $T_{\mathrm{g}}\left({ }^{\circ} \mathrm{C}\right)$ & $\Delta C_{\mathrm{p}}\left(\mathrm{J} \cdot \mathrm{g}^{-1} \cdot \mathrm{K}^{-1}\right)$ of confiner polymer & $\%$ of confiner polymer in PLLA/confiner films $\Delta C_{\mathrm{p}} / \Delta C_{\mathrm{p}, \text { bulk }}$ \\
\hline PS bulk & $97 \pm 1$ & $0.20 \pm 0.01$ & 100 \\
\hline PS/PLLA $300 \mathrm{~nm}$ amorphous & $97 \pm 1$ & $0.16 \pm 0.01$ & $78 \pm 5$ \\
\hline PS/PLLA $300 \mathrm{~nm}$ annealed $85^{\circ}$ & $98 \pm 1$ & $0.18 \pm 0.02$ & $90 \pm 10$ \\
\hline PS/PLLA $20 \mathrm{~nm}$ amorphous & $97 \pm 1$ & $0.15 \pm 0.01$ & $75 \pm 5$ \\
\hline PS/PLLA $20 \mathrm{~nm}$ annealed $85{ }^{\circ} \mathrm{C}$ & $98 \pm 1$ & $0.15 \pm 0.01$ & $75 \pm 5$ \\
\hline PC bulk & $144 \pm 1$ & $0.24 \pm 0.01$ & 100 \\
\hline PC/PLLA $300 \mathrm{~nm}$ amorphous & $144 \pm 1$ & $0.19 \pm 0.03$ & $78 \pm 10$ \\
\hline PC/PLLA $20 \mathrm{~nm}$ amorphous & $142 \pm 1$ & $0.14 \pm 0.02$ & $60 \pm 10$ \\
\hline PC/PLLA $300 \mathrm{~nm}$ annealed $85{ }^{\circ} \mathrm{C}$ & $144 \pm 1$ & $0.13 \pm 0.03$ & $52 \pm 10$ \\
\hline PC/PLLA $300 \mathrm{~nm}$ annealed $120^{\circ} \mathrm{C}$ & $145 \pm 1$ & $0.17 \pm 0.03$ & $73 \pm 10$ \\
\hline PC/PLLA $20 \mathrm{~nm}$ annealed $85^{\circ} \mathrm{C}$ & $142 \pm 1$ & $0.17 \pm 0.02$ & $70 \pm 10$ \\
\hline PC/PLLA $20 \mathrm{~nm}$ annealed $120{ }^{\circ} \mathrm{C}$ & $140 \pm 1$ & $0.17 \pm 0.02$ & $72 \pm 10$ \\
\hline
\end{tabular}

Table 4. Quantification of the Three-Phase Model of PLLA in PS/PLLA and PC/PLLA Multinanolayer Films ${ }^{a}$

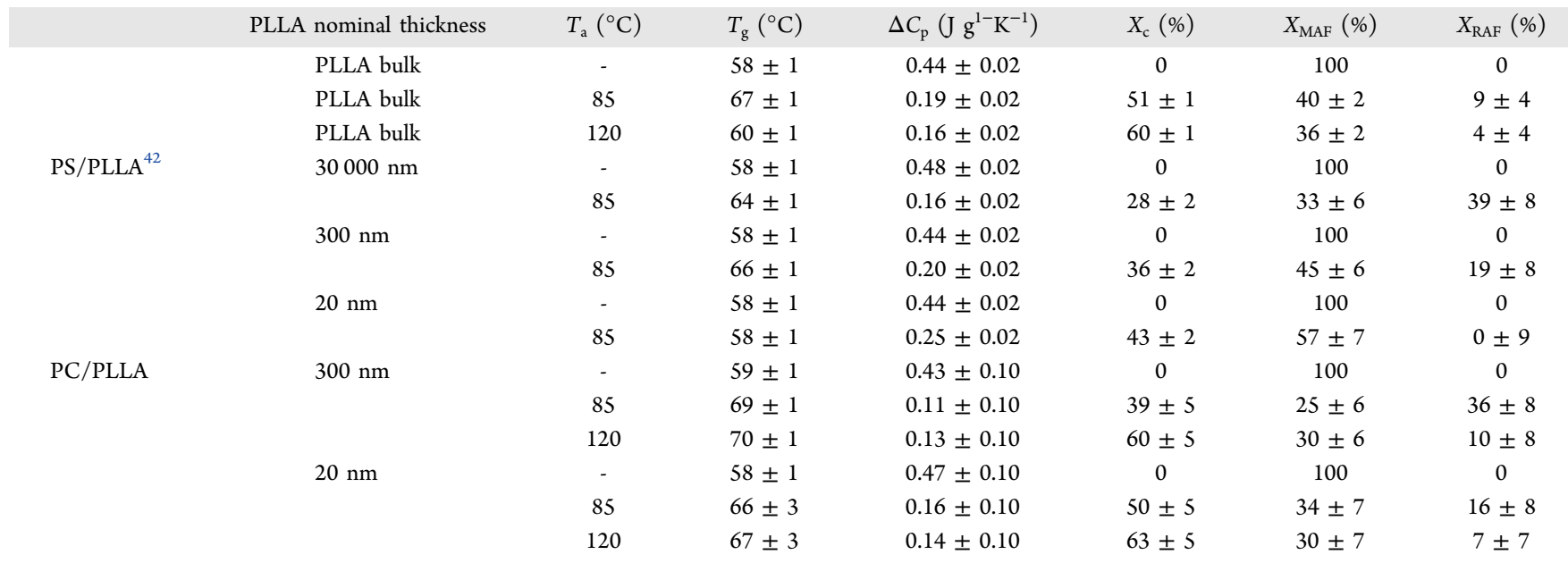

${ }^{a} T_{\mathrm{a}}$ annealing temperature at constant annealing time $=180 \mathrm{~min}, T_{\mathrm{g}}$ PLLA glass transition temperature, $\Delta C_{\mathrm{p}}$ normalized heat capacity step at $T_{\mathrm{g}}$ to $25 \%$ PLLA, $X_{\mathrm{c}}$ crystallinity degree, $X_{\mathrm{MAF}}$ quantity of mobile amorphous fraction, $X_{\mathrm{RAF}}$ quantity of rigid amorphous fraction. Data of the PS/PLLA systems were published in our earlier work, ref 42.

measured quantities were normalized to the PLLA content of 25 wt \%.

The amount of crystallinity of PLLA is given in Table 4. The results of the PS/PLLA system were discussed in our earlier work. $^{42}$ We show them here (Figure 6e) for the sake of comparison. The most important conclusion presented in ref 42 says that the confinement of PLLA between noninteracting (slippery) PS walls allowed for crystallizing PLLA with decoupling of amorphous and crystalline phase (0\% RAF in the crystallized $20 \mathrm{~nm}$ thick films, Table 4). In the confined layers, the RAF did not develop in parallel to the growth of PLLA crystals. This constitutes an important difference to bulk materials, where both phases were shown to develop at the same time. $^{47}$

In the case of sticky walls in the PC/PLLA system, the quantification of the different phases was difficult because of very small signals. It can be observed in Figure 6f, that the glass transition signal of PLLA after annealing almost disappeared. In these conditions, the quantification of MAF and RAF was done using signals obtained from the heat-only protocol (for details, see Supporting Information S.3). However, this calculation cannot consider the possible existence of an interphase between PC and PLLA. The data presented in Table 4 are therefore submitted to high variability. The final crystallinity degree of PLLA crystallized at $120{ }^{\circ} \mathrm{C}$ was higher than the one obtained at $85{ }^{\circ} \mathrm{C}$ regardless of the layer thickness. This is coherent with established crystallization kinetics of PLLA and with the results obtained by WAXS analysis. Independently of both the layer thickness and the crystallization conditions, the $T_{\mathrm{g}}$ of PLLA clearly shifted to higher temperatures with annealing, showing that the amorphous dynamics are hindered by the adjacent crystalline lamellas. As shown in Table 4, when PLLA crystallizes against PS without forming RAF, no variation of $T_{\mathrm{g}}$ was recorded (see results for $20 \mathrm{~nm}$ layer thickness). Therefore, one can expect that the RAF contributes to the mobility restrictions in the amorphous phase of PLLA when crystallized in multilayer films. Here, we cannot clearly evidence an impact of the crystallization temperature on the RAF formation because of the high variability of data. Current literature data including our own work indicate that RAF contents at lower crystallization temperature should be higher, because at low temperature, the hindered mobility of polymer segments affords more coupling points between crystalline and amorphous phase. As a consequence, geometrical limitations increase, which maximizes RAF formation. ${ }^{4,42,47}$ Despite the uncertainties, it seems that the RAF development in PC/PLLA is independent from the PLLA layer thickness and that more $\mathrm{RAF}$ is created at lower temperature. This is the contrary to the results of the PS/PLLA system reported for the annealed 20 

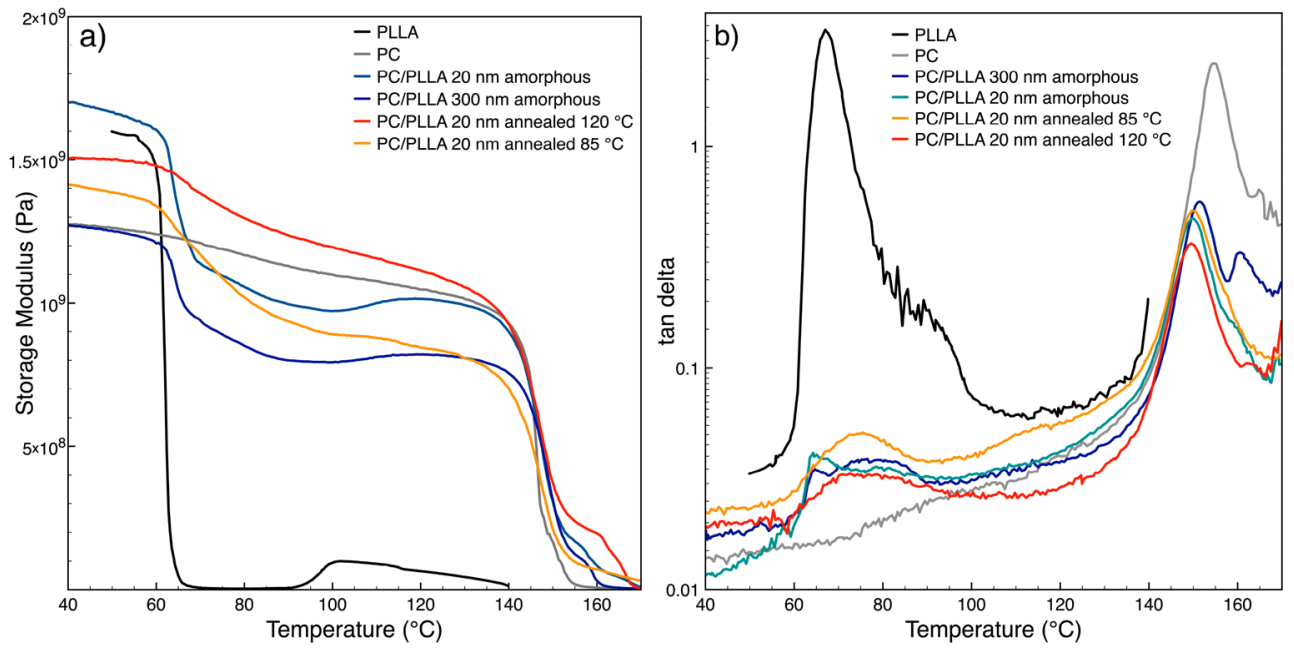

Figure 7. Storage modulus (a) and damping factor (b) of PC and PLLA bulk and amorphous and annealed (85 and $\left.120{ }^{\circ} \mathrm{C}\right)$ PC/PLLA at 300 and $20 \mathrm{~nm}$.

nm thick PLLA layers. We conclude that the interaction of PC walls with PLLA did not allow for the decoupling of the amorphous and crystalline phase when the annealing was carried out at $85{ }^{\circ} \mathrm{C}$.

Dynamic Mechanical Analysis of PC/PLLA. Because of the very small signal of the PLLA glass transition in the PC/ PLLA system in MT-DSC leading to high variability, DMA measurements were carried out (Figure 7).

Figure $7 \mathrm{a}$ shows the storage modulus $\mathrm{E}^{\prime}$ of PC/PLLA as a function of temperature. In the aim to magnify differences, a linear scale of $\mathrm{E}^{\prime}$ is used. PLLA alone shows an important decrease of the elastic modulus upon glass transition and then an increase around $100{ }^{\circ} \mathrm{C}$ due to cold crystallization. The multilayer films with PC had higher elastic modulus in the rubbery plateau because $\mathrm{PC}$ is in its glassy state. The glass transition of amorphous PLLA in the multilayer films is sudden. It is more gradual but still visible when PLLA was recrystallized. This may correspond to the progressive devitrification of RAF and/or PC/PLLA interphase. Figure $7 \mathrm{~b}$ shows the $\tan \delta$ curves of PC/PLLA multinanolayered films. It is possible to observe that the $\tan \delta$ peak of PC shifted to lower temperatures in the presence of PLLA, which can be attributed to the compatibility between PC and PLLA. In the literature, the appearance of an interphase between PC and PLLA in polymer blends was described and attributed to a transesterification reaction. ${ }^{61,62}$ Liu et al. ${ }^{63}$ studied the transesterification mechanism between PLA and PC under flow field by adding catalyst to the PLA/PC blend. They found that the transesterification reaction between PLA and PC could occur even without catalyst.

Impact of PLLA Confinement on Oxygen Barrier Properties. Table 5 reports the oxygen permeability of PS/ PLLA and PC/PLLA films. The multilayer films containing PLLA had better barrier properties than the PS or PC films, behavior which was expected because PLLA has lower permeability to $\mathrm{O}_{2}$ than PS and PC. The gain in overall barrier properties of the different systems compared with the properties of the confiner polymer is reported in Table 5. The fully amorphous systems showed an increase in $\mathrm{O}_{2}$ barrier properties by a factor 2, when PLLA was combined with PS or PC. The crystallization of the PLLA layers permitted gains up to a factor 12 for PS and to a factor 8 for PC.
Table 5. Oxygen Permeability of PS/PLLA and PC/PLLA Films and the Gain of Barrier Properties with Respect to the Confiner Polymer

\begin{tabular}{|c|c|c|c|c|c|}
\hline & $\begin{array}{l}\text { PLLA nominal } \\
\text { thickness }\end{array}$ & $\begin{array}{l}T_{\mathrm{a}} \\
\left({ }^{\circ} \mathrm{C}\right)\end{array}$ & $\begin{array}{c}t_{\mathrm{a}} \\
(\mathrm{min})\end{array}$ & $\begin{array}{c}P\left(\mathrm{O}_{2}\right) \times 10^{-18} \\
\left(\mathrm{~m}^{3} \cdot \mathrm{m} \cdot \mathrm{m}^{-2} \cdot \mathrm{s}^{-1} \cdot \mathrm{Pa}^{-1}\right)\end{array}$ & gain $^{a}$ \\
\hline \multirow{7}{*}{$\begin{array}{l}\text { PS/ } \\
\text { PLLA }\end{array}$} & PS bulk & & & $15.3 \pm 0.2$ & \\
\hline & PC bulk & & & $10.1 \pm 0.1$ & \\
\hline & PLLA bulk & - & - & $2.4 \pm 0.5$ & \\
\hline & PLLA bulk & 85 & 180 & $2.2 \pm 0.3$ & \\
\hline & PLLA bulk & 120 & 180 & $0.43 \pm 0.01$ & \\
\hline & $300 \mathrm{~nm}$ & - & - & $7.8 \pm 0.8$ & 2 \\
\hline & $300 \mathrm{~nm}$ & 85 & 180 & $4.2 \pm 0.5$ & 3 \\
\hline \multirow{9}{*}{$\begin{array}{l}\text { PC/ } \\
\text { PLLA }\end{array}$} & $20 \mathrm{~nm}$ & - & - & $6.6 \pm 0.3$ & 2 \\
\hline & $20 \mathrm{~nm}$ & 85 & 180 & $1.3 \pm 0.2$ & 12 \\
\hline & $20 \mathrm{~nm}$ & 100 & 180 & $12.6 \pm 0.8$ & 1.2 \\
\hline & $300 \mathrm{~nm}$ & - & - & $5.6 \pm 0.7$ & 2 \\
\hline & $300 \mathrm{~nm}$ & 85 & 180 & 11 & none \\
\hline & $300 \mathrm{~nm}$ & 120 & 180 & $1.3 \pm 0.1$ & 8 \\
\hline & $20 \mathrm{~nm}$ & - & - & $4.6 \pm 0.3$ & 2 \\
\hline & $20 \mathrm{~nm}$ & 85 & 180 & $6.5 \pm 0.5$ & 1.5 \\
\hline & $20 \mathrm{~nm}$ & 120 & 180 & $1.3 \pm 0.1$ & 8 \\
\hline \multicolumn{6}{|c|}{${ }^{a}$ gain $=\left(P_{\text {confiner }} / P_{\text {multilayer }}\right)$} \\
\hline
\end{tabular}

To investigate the property change of the confined PLLA layers, we used the law of serial resistances (eq 2) for the calculation of the permeability of PLLA. The implicit hypothesis is that the barrier properties of the confiner polymers PS and PC are constant. This hypothesis is commonly used in the study of multilayer films. ${ }^{19,22}$

Figure 8 shows the whole permeability data set in the aim to picture the evolution of the barrier properties with changing PLLA microstructure (both crystalline and amorphous phases). The exact conditions and numerical data of each point are shown in the Supporting Information S.5.

Amorphous Samples. The inspection of PS/PLLA data shows that the PLLA barrier properties of the amorphous samples were equal to the bulk data, regardless of the PLLA layer thickness and processing conditions. The obtained values correspond to usually reported data. ${ }^{2}$ The barrier properties of PLLA layers of $300 \mathrm{~nm}$ thickness between PC layers were equal to the bulk value. Interestingly, a small decrease in $\mathrm{P}\left(\mathrm{O}_{2}\right)$ 


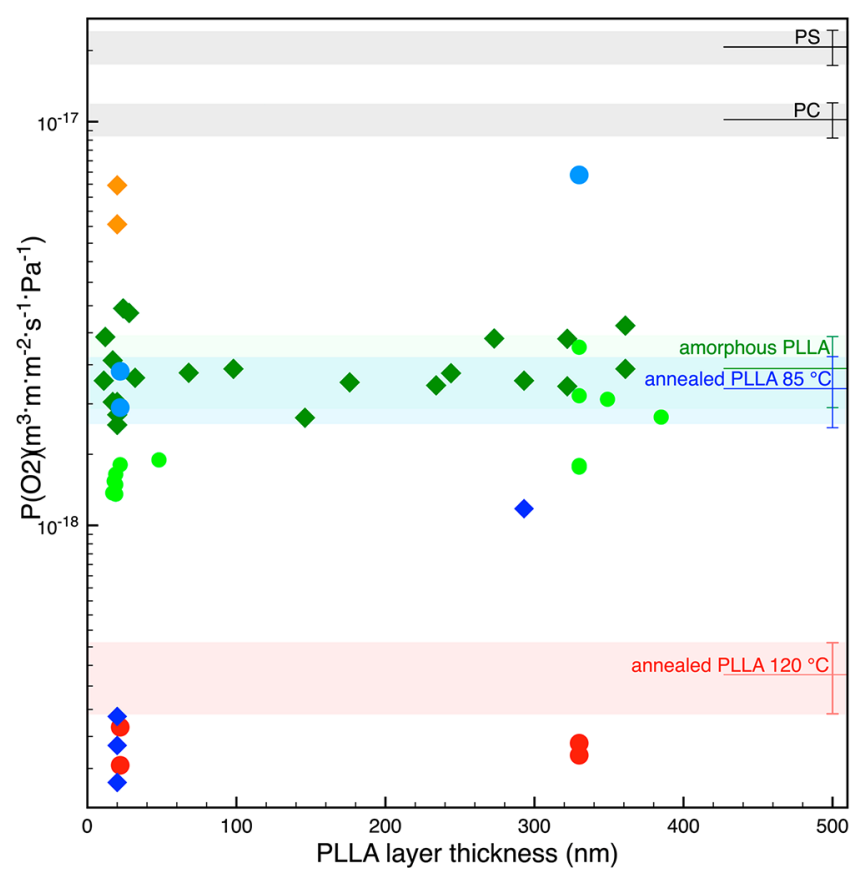

Figure 8. Oxygen permeability of PLLA in PS/PLLA ( $)$ and PC/ PLLA (-) films in comparison to the permeability data of the bulk materials (annealing time $180 \mathrm{~min}$ ). The annealing conditions are distinguished by the color of the symbols: amorphous PLLA green $\downarrow$, light-green 9 ; annealed PLLA at $85^{\circ} \mathrm{C}$ for 180 min between PS layers (blue $\bullet$ ), between PC layers (light-blue $\bullet$ ); annealed PLLA at 100 ${ }^{\circ} \mathrm{C}$ for $180 \mathrm{~min}$ between PS layers (orange $)$ ), annealed at $120^{\circ} \mathrm{C}$ for 180 min between PC layers (red 0 ). The value of the bulk materials is indicated as a colored range corresponding to the variability interval. It used the properties measured in the actual study and the ones measured in our earlier study (ref 4 ).

was observed at $20 \mathrm{~nm}$ layer thickness. This might be an effect of the interface compatibility.

Annealed PS/PLLA Films. The more interesting results are obtained after the annealing of the PLLA layers. The $P\left(\mathrm{O}_{2}\right)$ of the annealed $300 \mathrm{~nm}$ layers was lower than the one of the annealed bulk sample. Surprisingly, the $P\left(\mathrm{O}_{2}\right)$ of PLLA in 20 $\mathrm{nm}$ thick layers was lowered by 1 order of magnitude compared with the bulk and even better than the results obtained with high-temperature annealing, although the crystallinity degree was lower (43 vs $60 \%$, Table 4 ), and no homogeneous orientation of crystalline lamellas perpendicular to the gas permeation direction was obtained (Figure 4). The PS/PLLA-20 nm sample showed crystallization kinetics without secondary crystallization, changed Avrami exponents, and most importantly, no occurrence of RAF within the experimental uncertainty (Table 2 and 4, ref 42). We conclude therefore that the change in crystalline organization leading to a negligible amount of RAF caused the important improvement of PLLA barrier properties. An interesting result for choosing annealing process parameters of confined PLLA was obtained using a treatment at $100{ }^{\circ} \mathrm{C}$. This temperature was chosen in the aim to obtain a different PLLA polymorph, but it fell in the glass transition region of the confiner polymer. In that case, the barrier properties decreased, most probably due to layer breakup (see Figure 1 and Supporting Information S.5, Table S.4). A conclusion is that the confiner polymer needs to be glassy during the annealing treatment to be able to stabilize the structure.
Annealed PC/PLLA Films. PC/PLLA had a different behavior than PS/PLLA. The PLLA layers annealed at 85 ${ }^{\circ} \mathrm{C}$ did not show any increase of barrier properties compared to the bulk, although the crystalline orientation in the $20 \mathrm{~nm}$ thick films was in-plane. Table 4 shows that there the quantity of RAF in PC/PLLA annealed at $85{ }^{\circ} \mathrm{C}$ was superior to the bulk sample. In particular, it was very high in the PC/PLLA$300 \mathrm{~nm}$ films which had also high permeability (Figure 8). Moreover, the RAF quantity in the PC/PLLA-20 nm was apparently higher than in PS/PLLA. This can be observed looking at the very broad glass transition (Figure 6), although the quantitative determination was subject to uncertainty (Table 4). This is a clear impact of the properties of the confiner polymer and a possible interphase. This result shows again that the crystalline in-plane orientation alone was not enough to increase the barrier properties, but that the RAF is detrimental to the barrier properties of the PC/PLLA crystallized at $85{ }^{\circ} \mathrm{C}$. It could be opposed to this reasoning that the annealing treatment at $120{ }^{\circ} \mathrm{C}$ yielded a substantial gain in barrier properties (Figure 8), although RAF formed in PC/PLLA when crystallizing at $120^{\circ} \mathrm{C}$. It is worth mentioning that the percentage of RAF was lower compared with the treatment at $85^{\circ} \mathrm{C}$, even if variability was high. Moreover, the observed permeability values were hardly lower than what can be obtained by cold crystallization of the bulk at high temperature without RAF. Our hypothesis to explain this result is that the crystallites, despite the high crystallinity degree and in-plane orientation (Figure 5), might not overlap enough or be large enough to induce an important tortuosity on the diffusive pathway. It is also interesting to question whether the RAF could differently impact the barrier properties depending on its temperature of formation. The temperature of RAF devitrification, which ranges between the glass transition and the melting temperature, indicates its level of ordering. ${ }^{47}$ In bulk conditions, the stack model can be used to describe the organization of crystalline lamellas and the location of RAF. Righetti et al. ${ }^{64}$ proposed that the PLA crystallites can be described with the heterogeneous stack model as its glass transition increases with decreasing the crystallization temperature. This implies that the RAF is selectively positioned between lamellas and MAF between the stacks. As previously mentioned, the change in the Avrami parameters when confining PLLA in the multilayer structure suggests a different crystalline organization. A hypothesis would be that in the case of nonoverlapping lamellas, RAF adjacent to the crystalline phase does impact notably on the diffusive pathway. In the PC/PLLA multilayer films crystallized at 85 and $120^{\circ} \mathrm{C}$, the uncertainties around the glass transition are too high to discuss its evolution with the crystallization temperature. To summarize, the negative impact of the RAF on barrier properties is clearly assessed, but a quantitative relationship cannot be given today.

\section{CONCLUSIONS}

The innovative layer multiplying coextrusion process allowed the fabrication of multilayered films composed of alternating layers of PLLA and an amorphous polymer (PS or PC). Continuous layers with individual thicknesses of PLLA down to $20 \mathrm{~nm}$ were obtained. The study of those films extended our insights into PLLA crystallization under confinement. In particular, by choosing two amorphous polymers (PS and PC) having different chemical affinities with PLLA, the role of the confining polymer was investigated. 
While the as-extruded films contained amorphous PLLA layers, high degrees of crystallinity were achieved by thermal annealing at temperatures below the glass transition of the confining polymer. Highly orientated lamellas were obtained having in-plane orientation in the PC/PLLA samples and mixed in-plane and on-edge orientation in the PS/PLLA samples. This suggests that a higher interaction between the two polymers can favor the formation of in-plane lamellas. The quantitative study of the amorphous and crystalline phases in PS/PLLA and PC/PLLA films showed that decoupling of amorphous and crystalline phases characterized by low RAF during annealing at low temperature was only possible when the confiner polymer had low interaction with the PLLA layers. The occurrence of RAF in PC/PLLA samples had a negative impact on the barrier properties of the films, which could not be attenuated by the presence of in-plane crystals. More interestingly, annealing PS/PLLA films to obtain a high crystallinity degree without RAF allowed for a barrier improvement of the PLLA layers by a factor of 10 compared with amorphous PLLA. This was 2 times better than semicrystalline bulk PLLA. This unique result informs on the impact of the phase coupling on the PLLA barrier properties and on what properties can be probably achieved by nonoriented crystallization of PLA.

The exact processing parameters to obtain the mulitnanolayer films (S.1), the analysis of the viscosity ratio of PC and PLLA (S.2), MT-DSC protocols with an example of data treatment (S.3), supplementary AFM pictures (S.4), numerical data of the oxygen permeability of each sample used in Figure 8 (S.5) (PDF)

\section{AUTHOR INFORMATION}

\section{Corresponding Author}

Sandra Domenek - Universite Paris-Saclay, AgroParisTech, INRAE, UMR 0782 SayFood, 91300 Massy, France; ○ orcid.org/0000-0003-3012-041X;

Email: sandra.domenek@agroparistech.fr

\section{Authors}

Samira Fernandes Nassar - Universite Paris-Saclay, AgroParisTech, INRAE, UMR 0782 SayFood, 91300 Massy, France

Nicolas Delpouve - Normandie Univ, UNIROUEN Normandie, INSA Rouen, CNRS, Groupe de Physique des Materiaux, 76000 Rouen, France; (1) orcid.org/0000-00026064-7151

Cyrille Sollogoub - Laboratoire PIMM, Arts et Métiers, CNRS, CNAM, Hesam Université, F-75013 Paris Cedex, France; (1) orcid.org/0000-0003-2204-3696

Alain Guinault - Laboratoire PIMM, Arts et Metiers, CNRS, CNAM, Hesam Universite, F-75013 Paris Cedex, France

Gregory Stoclet - Univ Lille, CNRS, INRA, ENSCL, UMR 8207 - UMET - Unité Materiaux et Transformations, F-59000 Lille, France; (1) orcid.org/0000-0003-1510-0234

Gilles Régnier - Laboratoire PIMM, Arts et Métiers, CNRS, CNAM, Hesam Université, F-75013 Paris Cedex, France

\section{Notes}

The authors declare no competing financial interest.

\section{ACKNOWLEDGMENTS}

The authors acknowledge the Ph.D. grant CAPES n ${ }^{\circ}$ 9712-13-5 for Samira Fernandes Nassar. Furthermore, they acknowledge the technical help of Flavien Lecourtier (INRAE) for the measurement of the oxygen permeability.

\section{REFERENCES}

(1) Ducruet, V.; Domenek, S. Characteristics and Applications of Poly(lactic acid). In Biodegradable and Bio-Based Polymers: Environmental and Biomedical Applications, Kalia, S.; Averous, L., Eds.; Scrivener Publishing LLC: Beverly, MA, 2015; Chapter 6, pp 171224.

(2) Sonchaeng, U.; Iniguez-Franco, F.; Auras, R.; Selke, S.; Rubino, M.; Lim, L. T. Poly(lactic Acid) Mass Transfer Properties. Prog. Polym. Sci. 2018, 86, 85-121.

(3) Guinault, A.; Sollogoub, C.; Ducruet, V.; Domenek, S. Impact of Crystallinity of Poly(lactide) on Helium and Oxygen Barrier Properties. Eur. Polym. J. 2012, 48 (4), 779-788.

(4) Nassar, S. F.; Guinault, A.; Delpouve, N.; Divry, V.; Ducruet, V.; Sollogoub, C.; Domenek, S. Multi-Scale Analysis of the Impact of Polylactide Morphology on Gas Barrier Properties. Polymer 2017, 108, 163-172.

(5) Cocca, M.; Di Lorenzo, M. L.; Malinconico, M.; Frezza, V. Influence of Crystal Polymorphism on Mechanical and Barrier Properties of Poly(L-lactic Acid). Eur. Polym. J. 2011, 47, 1073-1080.

(6) Drieskens, M.; Peeters, R.; Mullens, J.; Franco, D.; Lemstra, P. J.; Hristova-Bogaerds, D. G. Structure Versus Properties Relationship of Poly(lactic Acid). I. Effect of Crystallinity on Barrier Properties. J. Polym. Sci., Part B: Polym. Phys. 2009, 47 (22), 2247-2258.

(7) Courgneau, C.; Domenek, S.; Lebosse, R.; Guinault, A.; Averous, L.; Ducruet, V. Effect of Crystallization on Barrier Properties of Formulated Polylactide. Polym. Int. 2012, 61 (2), 180-189.

(8) Colomines, G.; Ducruet, V.; Courgneau, C.; Guinault, A.; Domenek, S. Barrier Properties of Poly(lactic Acid) and its Morphological Changes Induced by Aroma Compound Sorption. Polym. Int. 2010, 59 (6), 818-826.

(9) Delpouve, N.; Stoclet, G.; Saiter, A.; Dargent, E.; Marais, S. Water Barrier Properties in Biaxially Drawn Poly(lactic Acid) Films. J. Phys. Chem. B 2012, 116 (15), 4615-4625.

(10) Boufarguine, M.; Guinault, A.; Miquelard-Garnier, G.; Sollogoub, C. PLA/PHBV Films with Improved Mechanical and Gas Barrier Properties. Macromol. Mater. Eng. 2012, 298 (10), 10651073.

(11) Picard, E.; Espuche, E.; Fulchiron, R. Effect of an OrganoModified Montmorillonite on PLA Crystallization and Gas Barrier Properties. Appl. Clay Sci. 2011, 53 (1), 58-65.

(12) Carr, J. M.; Langhe, D. S.; Ponting, M. T.; Hiltner, A.; Baer, E. Confined Crystallization in Polymer Nanolayered Films: A Review. J. Mater. Res. 2012, 27 (10), 1326-1350.

(13) Wang, H.; Keum, J. K.; Hiltner, A.; Baer, E. Confined Crystallization of PEO in Nanolayered Films Impacting Structure and Oxygen Permeability. Macromolecules 2009, 42 (18), 7055-7066.

(14) Wang, H.; Keum, J. K.; Hiltner, A.; Baer, E.; Freeman, B.; Rozanski, A.; Galeski, A. Confined Crystallization of Polyethylene Oxide in Nanolayer Assemblies. Science 2009, 323 (5915), 757-760.

(15) Zhang, G. J.; Baer, E.; Hiltner, A. Gas Permeability of Poly(4Methylpentene-1) in a Confined Nanolayered Film System. Polymer 2013, 54 (16), 4298-4308.

(16) Ponting, M.; Lin, Y. J.; Keum, J. K.; Hiltner, A.; Baer, E. Effect of Substrate on the Isothermal Crystallization Kinetics of Confined Poly(Epsilon-Caprolactone) Nano Layers. Macromolecules 2010, 43 (20), 8619-8627. 
(17) Langhe, D. S.; Hiltner, A.; Baer, E. Melt Crystallization of Syndiotactic Polypropylene in Nanolayer Confinement Impacting Structure. Polymer 2011, 52 (25), 5879-5889.

(18) Yu, F. L.; Deng, H.; Bai, H. W.; Zhang, Q.; Wang, K.; Chen, F.; $\mathrm{Fu}, \mathrm{Q}$. Confine Clay in an Alternating Multi Layered Structure through Injection Molding: A Simple and Efficient Route to Improve Barrier Performance of Polymeric Materials. ACS Appl. Mater. Interfaces 2015, 7 (19), 10178-10189.

(19) Carr, J. M.; Mackey, M.; Flandin, L.; Hiltner, A.; Baer, E. Structure and Transport Properties of Polyethylene Terephthalate and Poly(Vinylidene Fluoride-Co-Tetrafluoroethylene) Multilayer Films. Polymer 2013, 54 (6), 1679-1690.

(20) Zhang, G. J.; Xu, H.; Macinnis, K.; Baer, E. Crystallization of Linear Low Density Polyethylene under Two-Dimensional Confinement in High Barrier Blend Systems. Polymer 2014, 55 (26), 68536860.

(21) Zhang, G. J.; Lee, P. C.; Jenkins, S.; Dooley, J.; Baer, E. The Effect of Confined Spherulite Morphology of High-Density Polyethylene and Polypropylene on Their Gas Barrier Properties in Multilayered Film Systems. Polymer 2014, 55 (17), 4521-4530.

(22) Messin, T.; Marais, S.; Follain, N.; Chappey, C.; Guinault, A.; Miquelard-Garnier, G.; Delpouve, N.; Gaucher, V.; Sollogoub, C. Impact of of Water and Thermal Induced Crystallizations in A PC/ MXD6Multilayer Film on Barrier Properties. Eur. Polym. J. 2019, 111, $152-160$.

(23) Su, H. J.; Xue, J.; Cai, P. L.; Li, J.; Guo, S. Y. Structure and Oxygen-Barrier Properties of (Linear Low-Density Polyethylene/ Ethylene-Vinyl Alcohol Copolymer)/Linear Low-Density Polyethylene Composite Films Prepared by Microlayer Coextrusion. J. Appl. Polym. Sci. 2015, 132 (27), 42211.

(24) Delpouve, N.; Delbreilh, L.; Stoclet, G.; Saiter, A.; Dargent, E. Structural Dependence of The Molecular Mobility in The Amorphous Fractions of Polylactide. Macromolecules 2014, 47 (15), 5186-5197.

(25) Wang, Y.; Chan, C. M.; Ng, K. M.; Li, L. What Controls The Lamellar Orientation At The Surface of Polymer Films During Crystallization? Macromolecules 2008, 41 (7), 2548-2553.

(26) Michell, R. M.; Muller, A. J. Confined Crystallization of Polymeric Materials. Prog. Polym. Sci. 2016, 54-55, 183-213.

(27) Liu, Y. X.; Chen, E. Q. Polymer Crystallization of Ultrathin Films on Solid Substrates. Coord. Chem. Rev. 2010, 254 (9-10), 1011-1037.

(28) Prud'homme, R. E. Crystallization and Morphology of Ultrathin Films of Homopolymers and Polymer Blends. Prog. Polym. Sci. 2016, 54-55, 214-231.

(29) Wang, H. P.; Keum, J. K.; Hiltner, A.; Baer, E. Impact of Nanoscale Confinement on Crystal Orientation of Poly(Ethylene Oxide). Macromol. Rapid Commun. 2010, 31 (4), 356-361.

(30) Ma, Y.; Hu, W. B.; Hobbs, J.; Reiter, G. Understanding Crystal Orientation in Quasi-One-Dimensional Polymer Systems. Soft Matter 2008, 4 (3), 540-543.

(31) Hu, W. B.; Cai, T.; Ma, Y.; Hobbs, J. K.; Farrance, O.; Reiter, G. Polymer Crystallization Under Nano-Confinement of Droplets Studied by Molecular Simulations. Faraday Discuss. 2009, 143, 129141.

(32) Ma, Y.; Hu, W. B.; Reiter, G. Lamellar Crystal Orientations Biased by Crystallization Kinetics in Polymer Thin Films. Macromolecules 2006, 39 (15), 5159-5164.

(33) Zhang, R. C.; Huang, Z. H.; Sun, D.; Lu, A.; Zhong, M. L.; Fang, Z. X.; Ji, D. H.; Xiong, G. Y.; Wan, Y. Z. Crystallization of Poly(L-Lactide) in A Confined Space Between Polycarbonate Layers. J. Polym. Mater. 2018, 35 (2), 171-179.

(34) Wu, N. J.; Ding, M. C.; Li, C. W.; Yuan, Y.; Zhang, J. M. Lamellar Orientation and Crystallization Dynamics of Poly (L-lactic Acid) Thin Films Investigated by In-Situ Reflection Absorption Infrared Spectroscopy. J. Phys. Chem. B 2011, 115 (40), 1154811553.

(35) Maillard, D.; Prud'Homme, R. E. Crystallization of Ultrathin Films of Polylactides: From Chain Chirality to Lamellar Curvature and Twisting. Macromolecules 2008, 41, 1705-1712.
(36) Mohamed, A.; Gordon, S. H.; Biresaw, G. Poly(lactic Acid)/ Polystyrene Bioblends Characterized by Thermogravimetric Analysis, Differential Scanning Calorimetry, and Photoacoustic Infrared Spectroscopy. J. Appl. Polym. Sci. 2007, 106 (3), 1689-1696.

(37) Leung, B. O.; Hitchcock, A. P.; Brash, J. L.; Scholl, A.; Doran, A. Phase Segregation in Polystyrene-Polylactide Blends. Macromolecules 2009, 42 (5), 1679-1684.

(38) Sarazin, P.; Favis, B. D. Morphology Control in Co-Continuous Poly(L-Lactide)/Polystyrene Blends: A Route towards Highly Structured and Interconnected Porosity in Poly(L-Lactide) Materials. Biomacromolecules 2003, 4 (6), 1669-1679.

(39) Biresaw, G.; Carriere, C. J. Interfacial Tension of Poly(lactic Acid)/Polystyrene Blends. J. Polym. Sci., Part B: Polym. Phys. 2002, 40 (19), 2248-2258.

(40) Imre, B.; Renner, K.; Pukanszky, B. Interactions, Structure and Properties in Poly(lactic acid)/Thermoplastic Polymer Blends. eXPRESS Polym. Lett. 2014, 8 (1), 2-14.

(41) Fang, H.; Zhang, Y.; Bai, J.; Wang, Z.; Wang, Z. Bimodal Architecture and Rheological and Foaming Properties for GammaIrradiated Long-Chain Branched Polylactides. RSC Adv. 2013, 3 (23), 8783-8795.

(42) Nassar, S. F.; Domenek, S.; Guinault, A.; Stoclet, G.; Delpouve, N.; Sollogoub, C. Structural and Dynamic Heterogeneity in the Amorphous Phase of Poly(L,L-Lactide) Confined at the Nanoscale by the Coextrusion Process. Macromolecules 2018, 51 (1), 128-136.

(43) Bironeau, A.; Salez, T.; Miquelard-Garnier, G.; Sollogoub, C. Existence of a Critical Layer Thickness in PS/PMMA Nanolayered Films. Macromolecules 2017, 50 (10), 4064-4073.

(44) Bironeau, A.; Dirrenberger, J.; Sollogoub, C.; MiquelardGarnier, G.; Roland, S. Evaluation of Morphological Representative Sample Sizes for Nanolayered Polymer Blends. J. Microsc. 2016, 264 (1), 48-58.

(45) Reading, M.; Hourston, D. Theory and Practice of Modulated Temperature Differential Scanning Calorimetry; Springer: Berlin, 2006.

(46) Park, S. H.; Lee, S. G.; Kim, S. H. Isothermal Crystallization Behavior and Mechanical Properties of Polylactide/Carbon Nanotube Nanocomposites. Compos. Composites, Part A 2013, 46, 11-18.

(47) Righetti, M. C.; Tombari, E. Crystalline, Mobile Amorphous and Rigid Amorphous Fractions in Poly(L-lactic acid) by TMDSC. Thermochim. Acta 2011, 522 (1-2), 118-127.

(48) Saeidlou, S.; Huneault, M. A.; Li, H.; Park, C. B. Poly(lactic acid) Crystallization. Prog. Polym. Sci. 2012, 37, 1657-1677.

(49) Wasanasuk, K.; Tashiro, K. Crystal Structure and Disorder in Poly(L-lactic Acid) Delta Form (Alpha' Form) and the Phase Transition Mechanism to the Ordered Alpha Form. Polymer 2011, 52 (26), 6097-6109.

(50) Kawai, T.; Rahman, N.; Matsuba, G.; Nishida, K.; Kanaya, T.; Nakano, M.; Okamoto, H.; Kawada, J.; Usuki, A.; Honma, N.; Nakajima, K.; Matsuda, M. Crystallization and Melting Behavior of Poly (L-lactic Acid). Macromolecules 2007, 40 (26), 9463-9469.

(51) Zhang, J.; Tashiro, K.; Tsuji, H.; Domb, A. J. Disorder-ToOrder Phase Transition and Multiple Melting Behavior of Poly(LLactide) Investigated by Simultaneous Measurements of WAXD and DSC. Macromolecules 2008, 41 (4), 1352-1357.

(52) Di Lorenzo, M. L.; Cocca, M.; Malinconico, M. Crystal Polymorphism of Poly(L-lactic acid) and its Influence on Thermal Properties. Thermochim. Acta 2011, 522 (1-2), 110-117.

(53) Lin, Y. J.; Hiltner, A.; Baer, E. Nanolayer Enhancement of Biaxially Oriented Polypropylene Film for Increased Gas Barrier. Polymer 2010, 51 (24), 5807-5814.

(54) Yasuniwa, M.; Tsubakihara, S.; Iura, K.; Ono, Y.; Dan, Y.; Takahashi, K. Crystallization Behavior of Poly(L-lactic acid). Polymer 2006, 47 (21), 7554-7563.

(55) Stoclet, G.; Seguela, R.; Vanmansart, C.; Rochas, C.; Lefebvre, J. M. WAXS Study of the Structural Reorganization of SemiCrystalline Polylactide under Tensile Drawing. Polymer 2012, 53 (2), 519-528. 
(56) Cartier, L.; Okihara, T.; Ikada, Y.; Tsuji, H.; Puiggali, J.; Lotz, B. Epitaxial Crystallization and Crystalline Polymorphism of Polylactides. Polymer 2000, 41 (25), 8909-8919.

(57) Delpouve, N.; Saiter, A.; Mano, J.; Dargent, E. Cooperative Rearranging Region Size in Semi-Crystalline Poly(L-lactic Acid). Polymer 2008, 49, 3130-3135.

(58) Delpouve, N.; Arnoult, M.; Saiter, A.; Dargent, E.; Saiter, J. M. Evidence of Two Mobile Amorphous Phases in Semicrystalline Polylactide Observed from Calorimetric Investigations. Polym. Eng. Sci. 2014, 54 (5), 1144-1150.

(59) Righetti, M. C.; Gazzano, M.; Delpouve, N.; Saiter, A. Contribution of the Rigid Amorphous Fraction to Physical Ageing of Semi-Crystalline PLLA. Polymer 2017, 125, 241-253.

(60) Fischer, E.; Sterzel, H.; Wegner, G. Investigation of Structure of Solution Grown Crystals of Lactide Copolymers by Means of Chemical Reactions. Kolloid Z. Z. Polym. 1973, 251, 980-990.

(61) Bao, D. F.; Liao, X.; He, G. J.; Huang, E. B.; Yang, Q.; Li, G. X. Effects of Enhanced Compatibility by Transesterification on the Cell Morphology of Poly(lactic acid)/ Polycarbonate Blends Using Supercritical Carbon Dioxide. J. Cell. Plast. 2015, 51 (4), 349-372.

(62) Chen, Y.; Peng, Y.; Liu, W. Y.; Zeng, G. S.; Yang, J. H.; Yan, X. $\mathrm{H}$. The Effect of Various Catalyzers on Transesterification in Reactive Blending PC/PLA Blends. Adv. Mater. Res. 2013, 741, 24-27.

(63) Liu, C.; Lin, S.; Zhou, C.; Yu, W. Influence of Catalyst on Transesterification between Poly(lactic acid) and Polycarbonate under Flow Field. Polymer 2013, 54 (1), 310-319.

(64) Righetti, M. C.; Prevosto, D.; Tombari, E. Time and Temperature Evolution of the Rigid Amorphous Fraction and Differently Constrained Amorphous Fractions in PLLA. Macromol. Chem. Phys. 2016, 217 (18), 2013-2026. 